\title{
Splenectomy versus Partial Splenic Artery Embolization for Management of Hypersplenism
}

\author{
Mohammed Matar, ${ }^{1}$ MD, FACS; Mohammed Gamal, ${ }^{2}$ MD; Khaled Sharaf, ${ }^{3}$ MD \\ ${ }^{1}$ General Surgery Department, Ain Shams University, Egypt \\ ${ }^{2}$ Radiology Department, Ain Shams University, Egypt \\ ${ }^{3}$ General Surgery Department, Zagazig University, Egypt
}

Background: Hypersplenism remains a major problem in patients with chronic liver disease. For a long time splenectomy either open or laparoscopic was the definitive line of treatment for such conditions. However, sometimes the operation may be dangerous for some patients with poor liver condition and patients unfit for general anesthesia. So in the recent years partial splenic embolization (PSE) has been used as an alternative for splenectomy in such patients.

Patients and methods: This comparative prospective study was conducted on 28 patients, with thrombocytopenia secondary to hypersplenism as the main clinical presentation, admitted to El-Demerdash and Ain Shams University Specialized hospitals between January 2015 and January 2017. They were classified into 2 groups A and B, with 14 patients in each group. Group A patients were treated by splenectomy, with prior upper GIT endoscopy to determine the presence of esophageal varices to be banded or ligated, while group B patients were treated by partial splenic embolization (PSE) aiming at establishing occlusion of $50-70 \%$ of the distal branches of the splenic artery. The study compared both groups as regard the improvement of the blood indices, RBC, TWBC and platelet count, length of hospital stay, and postoperative complications.

Results: There was statistically significant difference among both groups as regards operative time, estimated blood loss, rate of blood transfusion and length of hospital stay. Multiple blood transfusions and blood loss was markedly increased in the splenectomy group. Postoperative complications were reported to be $78.6 \%$ in the splenectomy group, which was significantly higher than in the PSE group, $57.1 \%$ of patients. In all studied patients, all the indices of the blood count showed significantly increased postoperative values compared to the preoperative values at the one month follow up. The mean RBC count did not differ significantly between both study groups postoperatively, whereas the mean TWBC and platelet counts were significantly higher in the splenectomy group than in the PSE group $(p<0.05, p<0.01)$.

Conclusion: Both techniques are followed by marked improvement of the platelet count but improvement was much better after splenectomy. The study also presented PSE as a good therapeutic modality in patients with hypersplenism secondary to chronic liver disease with the advantages of being a simple procedure performed under local anesthesia with fewer post-procedure complications.

Key words: Splenectomy, partial splenic embolization, thrombocytopenia.

\section{Introduction}

Portal hypertension in the setting of cirrhosis commonly leads to splenomegaly. ${ }^{1}$ The pathogenesis of each hematologic deficiency in cirrhotic patients is multi-factorial in nature. Splenic sequestration, however, serves as a common link; and is a contributing factor in the development of thrombocytopenia, anemia, and leukopenia in cirrhotic patients. ${ }^{2}$

The traditional treatment method for hypersplenism secondary to cirrhosis is splenectomy, however, due to the potential disadvantages of severe, postoperative complications and the high rates of perioperative mortality after splenectomy, ${ }^{3,4}$ the clinical application of this traditional mode of treatment has been limited. In addition, splenectomy is often associated with an increased long-term risk of septic events ${ }^{5,6}$ particularly pneumococcal infections. In recent years, partial splenic embolization (PSE) has been shown to be an effective way to relieve hypersplenism. ${ }^{7-9}$ Ligation of the splenic artery (LSA) is also used in the treatment of hypersplenism due to other causes. ${ }^{10}$ Additionally, some cirrhotic patients may be poor surgical candidates, thus necessitating alternative approaches to splenectomy. PSE was first performed in the treatment of hypersplenism by Spigos et al in 1979. ${ }^{11}$ Soon, PSE gained popularity and served as a therapeutic option for cirrhotic patients with hypersplenism who were poor surgical candidates, ${ }^{12}$ 


\section{Patients and methods}

This prospective comparative study was conducted on 28 patients who were admitted to El-Demerdash and Ain-Shams University hospitals in the period from January 2015 to January 2017 with thrombocytopenia due to chronic liver disease associated with hypersplenism. All patients were subjected to detailed history, thorough physical examination, laboratory investigations (Complete blood count, liver function tests, renal function tests, bleeding profile, viral markers as HBsAg and HCVAb, HIVAb), abdominal ultrasonography to evaluate liver regarding to size, echogenicity, presence of focal lesions, portal vein diameter and patency, splenic size and echogenicity, colorcoded duplex scanning of the portal circulation, upper gastrointestinal endoscopy, and abdominal computed tomography (CT) scans with oral and intravenous contrast. Following evaluation, the patients were categorized into 2 equal groups, 14 patients in each group using the Excel Random Sample (7.0) computer program: group A patients were treated by open splenectomy with prior upper GIT endoscopy to determine the presence of esophageal varices to be banded or ligated. Group B patients were treated by PSE to establish occlusion of at least $50 \%$ of the distal branches splenic artery.

All the patients of each group were informed about the risks and benefits of their selected procedure and a written consent was obtained from each patient before the procedure.

Patients of both groups received pneumococcal, meningiococcal and hemophilus influenza vaccination two weeks before procedure or surgery. The patients of group A recieved platelet transfusion within 6 hours before surgery to elevate the platelet count and decrease bleeding tendency during the operation. Postoperatively, both groups received prophylactic antibiotics 6 hours before the procedure that included $1 \mathrm{gram}$ Ceftazidime, 1.5 grams of combined ampicillin-sulbactam and $0.5 \mathrm{gram}$ of metronidazole. Postoperatively, same regimen was continued every 12 hours for 5 days in both groups.

Two procedures were performed in this study, open splenectomy was performed by the surgical team and the PSE was performed by the interventional radiologist under local anesthesia.

\section{Techniques:}

\section{Splenectomy procedure:}

The operation was performed under general anesthesia with laparoscopic approach according to standard procedures.13,14 All the operations were performed by the same experienced surgeon and team. In the procedure, abdominal exploration was done to look for accessory spleens. Then, the splenocolic ligament, inferior pole vessels (splenorenal ligament), short gastric vessels (gastrosplenic ligament), and phrenic attachments (splenophrenic ligament) were divided. Subsequently, the splenic artery and vein were dissected and ligated at the hilum, patients received pharmacologic thromboprophylaxis in the form of Colpidogrel after splenectomy or PSE to avoid the risk for thromboembolism provided that the patient wasn't suffering from a hematologic bleeding disorder. ${ }^{15}$

\section{Partial splenic embolization (PSE):}

Under strict aseptic condition, PSE was performed according to a standard approach. ${ }^{16}$ Briefly, the femoral artery was punctured by a 5.0 French catheter (Cook, Bloomington, USA; Terumo, Tokyo, Japan) via the Seldinger approach. Preliminary splenic arterial angiography was obtained to determine the configuration of splenic artery and the location of pancreatic branches. The tip of the catheter was placed as distal as possible at the hilus of the spleen in order to avoid ectopic embolization, and embolization was performed using embolic agent suspended in an antibiotic solution (Gentamicin sulphate $16 \mathrm{mg}$ ) and contrast medium. The splenic infarction ratio was set at $50-70 \% .{ }^{17}$ During embolization, small amounts of contrast material were periodically injected through the catheter to monitor the flow distribution in the spleen. Immediately after each particle injection, post-embolization angiography was performed and the infarction rate was calculated (Figsures 1,2). Once a $50-70 \%$ ablation of splenic parenchyma was achieved, catheter was irrigated with saline and removed and the site of puncture was compressed for about 15 minutes. Post-embolization computed tomography was performed to document the extent of devascularization immediately after the procedure.

The embolic agent used in this study was polyvinyl alcohol (PVA) in contour particles (Boston Scientific, Natick, MA, USA). For most of our patients, one vial of PVA was sufficient to embolize at least $50 \%$ of the spleen parenchyma.

All patients remained in hospital until postembolization syndrome or any other significant complications disappeared. Post-PSE supportive care included appropriate intravenous fluids infusion, systemic prophylaxis with intravenous antibiotics, Ceftazidime ( $1 \mathrm{~g} / 12 \mathrm{~h}$ ) for 5 days, and adapted analgesic treatment with nonsteroidal anti-inflammatory drugs or morphine for at least 3 days. 
The precise infarction rate was calculated on CT examinations 2 weeks after PSE. Routine abdominal ultrasonography was performed at 30 days after PSE to exclude portal vein thrombosis.

If a splenic abscess was suspected (by prolonged high grade fever, persistent agonizing left hypochondrial pain, signs of toxemia or neutrophilia in $\mathrm{CBC}), \mathrm{CT}$ abdomen was done.

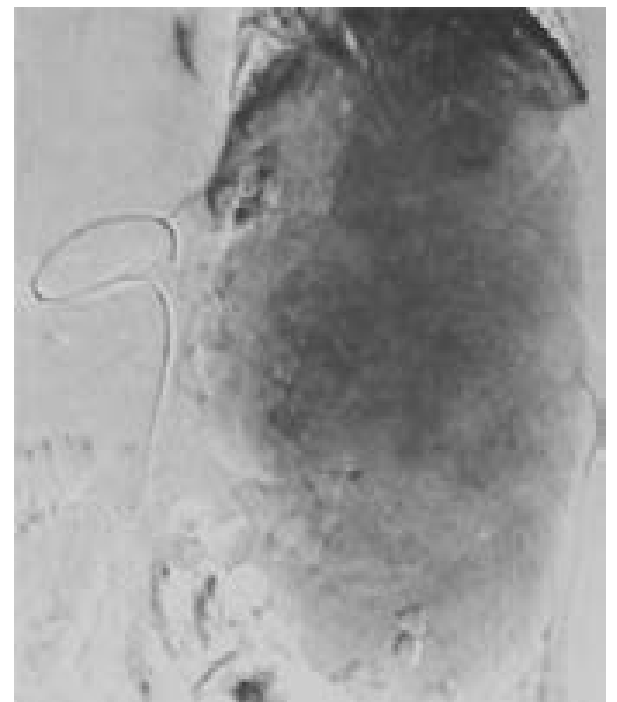

Fig 1: Splenic arteriogram, obtained before the procedure.

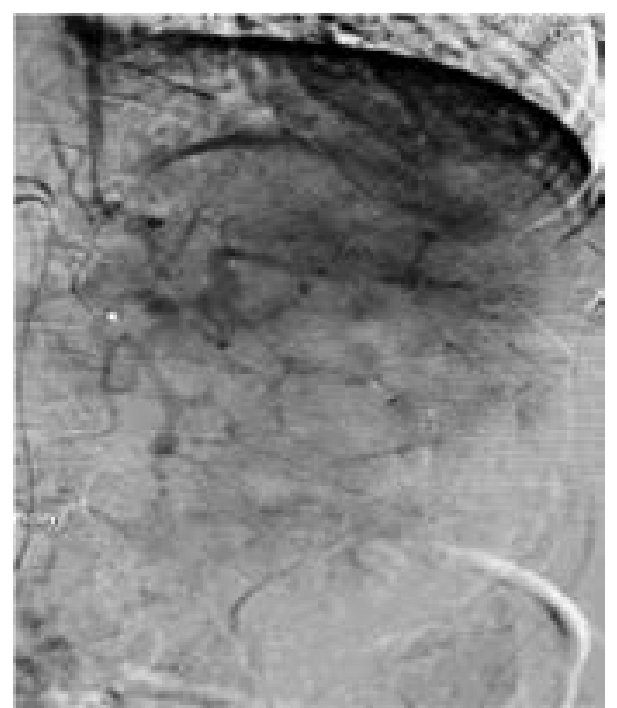

Fig 2: Splenic arteriogram, obtained after embolization, shows normal anatomy and parenchymal nonselective embolization shows abrupt occlusion enhancement pattern of many splenic arterial branches and patchy remnants of parenchymal blush.
Postoperative pain was scored using the numerical rating scale (NRS), with the rating of 1 out 5 meaning very mild pain and 5 out of 5 meaning severe pain. In both groups, the mean operative time, estimated blood loss at operation, length of hospital stay, need for blood transfusion and postoperative complications were recorded during follow up visits. Patients were seen after one month and then 6 months later. In the follow up visit, full clinical assessment, CBC, liver and kidney functions and abdominal ultrasound were done.

Statistical analysis: Continuous variables were expressed as mean \pm standard deviation (SD) and analyzed using -test. Qualitative variables were presented as number and percent. The chi-squared or Fischer's exact test was used for comparison between groups as appropriate, statistical analyses were performed using SPSS software (version 13.0; SPSS Inc., Chicago, IL), a value of $p<0.05$ was considered statistically significant.

\section{Results}

A total of 28 patients were included in the study, 16 males $(57.1 \%)$ and 12 females $(42.9 \%)$, and the mean patient age was $49.71 \pm 19.3$ years (range, 19-64 years). Group A had 9 males and 5 females. group $B$ patients included 7 males and 7 females. In both groups, comorbid illnesses included hypertension ( $n=6,21.4 \%)$, hyperlipidemia $(n=4$, $14.3 \%)$, diabetes mellitus $(n=9,32 \%)$, coronary artery disease $(n=4,14.3 \%)$, liver cirrhosis and/ or portal hypertension $(n=17,70.8 \%)$, renal dysfunction $(n=5,17.9 \%)$. They were reported in $78.6 \%$ of cases in group A versus $92.86 \%$ in group B patients. Chronic liver diseases and portal hypertension, as evaluated clinically and radiologically were $64.2 \%$ and $28.6 \%$ in group A versus $57 \%$ and $21.4 \%$ in group B. There was no statistically significant difference among both groups regarding the $C B C$ indices, mean $R B C$, TWBC and Platelet count (Table 1). 
Table 1: Demographics of study groups

\begin{tabular}{|c|c|c|c|}
\hline Parameters & $\begin{array}{c}\text { Splenectomy group A } \\
\text { (14) }\end{array}$ & PSE group B (14) & P-value \\
\hline Gender (M : F) & $9: 5$ & $7: 7$ & 0.852 \\
\hline Age (yr) & $42.1 \pm 8.7$ & $45.4 \pm 1.4$ & 0.671 \\
\hline RBC count $\left(10^{6} / \mathrm{mm}^{3}\right)$ & $2.1 \pm 1.72$ & $1.9 \pm 1.89$ & 0.528 \\
\hline TWBC count $\left(/ \mathrm{mm}^{3}\right)$ & $2.81 \pm 1.02$ & $3.19 \pm 0.95$ & 0.171 \\
\hline PLT count $\left(10^{3} / \mathrm{mm}^{3}\right)$ & $43.6 \pm 2.7$ & $37.5 \pm 2.4$ & 0.361 \\
\hline Spleen weight (g) & $1471 \pm 213$ & $1437 \pm 128$ & 0.436 \\
\hline Comorbidities & $11(78.6 \%)$ & $13(92.8 \%)$ & 0.751 \\
\hline Chronic Liver disease n (\%) & $9(64.2 \%)$ & $8(57 \%)$ & \\
\hline Portal hypertension n (\%) & $4(28.6 \%)$ & $3(21.4 \%)$ & \\
\hline Bleeding tendency $\mathrm{n}(\%)$ & $3(21.4 \%)$ & $4(28.6 \%)$ & \\
\hline Abnormal abdominal ultrasound $\mathrm{n}(\%)$ & $7(50 \%)$ & $11(78.6 \%)$ & \\
\hline
\end{tabular}

There was statistically significant difference among both groups as regards operative time, estimated blood loss, need for blood transfusion and length of hospital stay. Definitely, the procedure time in PSE group was much shorter than the surgical splenectomy $(24.13 \pm 5.52$ versus $107 \pm 12.3$ minutes, $p<0.05)$.

Multiple blood transfusions were needed in $35.7 \%$ of cases in the splenectomy group B, while no cases required blood transfusion in the PSE group $B$ patients $(P<0.05)$. Blood loss was marked in the splenectomy group with a mean of $720 \pm 129 \mathrm{ml}$ compared to minimal blood loss $(20 \pm 10 \mathrm{ml})$ in the PSE patients $(p<0.001)$. Patients in group $A$ had a longer hospital stay $(12.3 \pm 2.11$ versus $9.35 \pm 1.71$, $\mathrm{p}<0.001$ ) relative to group B patients (Table 2 ).

Table 2: Intraoperative and postoperative results

\begin{tabular}{lccc}
\hline Parameters & $\begin{array}{c}\text { Splenectomy group } \\
(\mathbf{1 4 )}\end{array}$ & PSE group (14) & P-value \\
\hline Operative time (min) & $107 \pm 12.3$ & $24.13 \pm 5.52$ & $<0.05$ \\
Estimated blood loss (mL) & $720 \pm 129$ & $20 \pm 10$ & $<0.001$ \\
No. of patients required blood transfusion & $5(35.71 \%)$ & 0 & $<0.05$ \\
$(\%)$ & & & $<0.001$ \\
Length of hospital stay (days) & $12.3 \pm 2.11$ & $9.35 \pm 1.71$ & $<0.001$ \\
Severe pain (\%) & $4(28.6 \%)$ & $11(78.6 \%)$ & 0.6839 \\
Complications total (\%) & $11(78.6 \%)$ & $6(43 \%)$ & \\
Intra-abdominal abscess & $1(7.1 \%)$ & $1(7.1 \%)$ & \\
Pleural effusion & $3(21.4 \%)$ & $1(7.1 \%)$ & \\
Pneumonia & $2(14.3 \%)$ & 0 & \\
Ascites & $7(50 \%)$ & $4(28.6 \%)$ & \\
Internal bleeding & $1(7.1 \%)$ & 0 & \\
Portal vein thrombosis & $1(7.1 \%)$ & 0 & \\
Liver Decompensation & 0 & $1(7.1 \%)$ & \\
\hline
\end{tabular}

Postoperative pain was the most common side effect and was treated with analgesics and severe pain responded to sedation, which gradually resolved by time. Postoperative severe pain (rating from 4-5 on the numerical rating scale) was reported in $28.6 \%$ of group A patients which was significantly lower than $78.6 \%$ of patients in group B $(p<0.001)$. Reported postoperative complications included one case of intra-abdominal abscess which occurred on the fifth postoperative day in the splenectomy group (7.1\%), and a splenic abscess that developed in the PSE group (7.1\%) after 10 days postoperatively, and both were treated by percutaneous drainage. A case of 
Intra-abdominal bleeding was reported in the splenectomy group, requiring exploration and evacuation, where the source of bleeding was the short gastric vessels and they were secured then the patient recovered satisfactorily. Pleural effusion occurred in $3(21.4 \%)$ patients in group $\mathrm{A}$ and $1(7.1 \%)$ patient in group B. Pneumonia was reported in $2(14.3 \%)$ of cases in the splenectomy group and was medically treated. One patient $(7.1 \%)$ developed portal vein thrombosis and was readmitted after 3 months with mesenteric vascular occlusion and resection anastomosis was done. Only one patient of the PSE group suffered from severe liver decompensation and was admitted to the intensive care unit and recovered after one month. No surgical site infections of mortalities were recorded in both groups. All performed PSE cases were successful to achieve the $50-70 \%$ occlusion of the splenic artery and achieved splenic infarction.

Table 3: Postoperative Laboratory results

\begin{tabular}{cccc}
\hline Parameters & Splenectomy group (14) & PSE group (14) & P-value \\
\hline RBC count $\left(10^{6} / \mathrm{mm}^{3}\right)$ & $4.46 \pm 0.51$ & $4.31 \pm 0.48$ & 0.315 \\
TWBC count $\left(/ \mathrm{mm}^{3}\right)$ & $7.314 \pm 2.516$ & $6.141 \pm 2.788$ & 0.05 \\
PLT count $\left(10^{3} / \mathrm{mm}^{3}\right)$ & $502,160 \pm 63.11$ & $491,362 \pm 81.30$ & 0.01 \\
\hline
\end{tabular}

In all studied patients, all the indices of the blood count showed significantly increased postoperative values compared to the preoperative values at the one month follow up. In the splenectomy group, the mean RBC increased significantly from $2.1 \pm 1.7210^{6} / \mathrm{mm}^{3}$ preoperative to $4.46 \pm$ $0.5110^{6} / \mathrm{mm}^{3}$ postoperative $(\mathrm{p}<0.05)$. The mean platelet count was $43.6 \pm 2.7 \mathrm{x} 10^{3} / \mathrm{mm}^{3}$ before the surgical procedure and changed significantly to $502.160 \pm 63.11 \times 10^{3} / \mathrm{mm}^{3} \quad(\mathrm{p}<0.001)$ after one month follow up. The white blood cell count showed significant improvement postoperatively, as the mean WBC was $4.36 \pm 1.72$ count $/ \mathrm{mm} 3$ before the surgical procedure and changed to $7.314 \pm 2.516$ count $/ \mathrm{mm}^{3}$ ( $\mathrm{p}$ value $<0.001$ ). Two cases in this group had to get hematologic consultation and started receiving anti-thrombotic medications as they experienced platelet counts higher than $800 \times 10^{3} / \mathrm{mm}^{3}$. PSE group also had significant increase in RBC, 4.31 $\pm 0.48 \times 10^{6} /$ $\mathrm{mm}^{3}$ post PSE compared to $1.9 \pm 1.89 \times 10^{6} / \mathrm{mm}^{3}$ preoperative values $(p<0.01)$. Again the platelet count increased significantly postoperatively than preoperative $\left(491,362 \pm 81.303 \times 10^{3} / \mathrm{mm}^{3}\right.$ versus $\left.37.5 \pm 2.47 \times 10^{3} / \mathrm{mm}^{3} \quad(p<0.001)\right)$. The TWBC showed significant rise from $3.19 \pm 0.95 /$ $\mathrm{mm} 3$ preoperatively to $6.141 \pm 2.788 / \mathrm{mm}^{3}$ postoperatively $(p<0.05)$. The mean RBC count did not differ significantly between both study groups postoperatively, whereas the mean TWBC and platelet counts were significantly higher in the splenectomy group than in the PSE group $(\mathrm{p}<$ $0.05, \mathrm{p}<0.01$ ).

\section{Discussion}

Splenectomy is the most common surgical treatment for massive splenomegaly and hyperspenism. ${ }^{14}$ It has been generally agreed that splenectomy is performed to control esophageal varices bleeding or as a modality in order to reverse severe thrombocytopenia and leukopenia. However, there are some risks associated with splenectomy ${ }^{6}$ such as hemorrhage, pulmonary atelectasis, pneumonia, pleural effusion, subphrenic abscess, gastric ileus, venous thrombosis, overwhelming postoperative infection (OPSI), and atherosclerosis. Alternatively, PSE could be an option that may have some advantages over splenectomy in some instances. ${ }^{17}$ Furthermore, PSE has been used to treat various conditions, which include chronic idiopathic thrombocytopenic purpura, hereditary spherocytosis, and also splenic trauma in haemodynamically unstable patients. PSE provides a minimally invasive alternative to splenectomy in patients who are severely compromised because of splenomegaly or sequestration and destroying in the spleen. ${ }^{18}$

In our study, we compared the two procedures of surgical splenectomy and interventional PSE as regards the complication rate and their effect on blood indices, namely, RBC, TWBC and platelet count.

Notably, the mean operative time, estimated blood loss, need for blood transfusion, severe pain, and postoperative length of hospital stay in the PSE group were significantly lower than the SP group. The total complication rate was similar between the splenectomy and PSE groups.

Postoperative RBC, WBC and Platelet counts rose significantly in the splenectomy and PSE groups compared with their preoperative results. The postoperative increases of WBC and PLT counts in the splenectomy group were higher than those in the PSE group. Our results are in agreement with those of Shoufei et al, $2016^{19}$ who concluded that both splenectomy and PSE could increase WBC and PLT counts, in patients with massive splenomegaly 
and possible prevention of disease progression by both procedures.

However, there is some concern regarding decreased portal-vein flow and a rapid increase in the platelet count after PSE which may induce portal-vein thrombosis. ${ }^{3}$ Moreover, the extent of embolization seems to be critical for long-term efficacy of PSE, embolization of less than $50 \%$ of the splenic mass was almost always associated with a relapse of hypersplenism or continuation of splenic activity. ${ }^{17}$

\section{Conclusion}

Partial splenic embolization is an effective therapeutic modality for the treatment of hypersplenism secondary to chronic liver disease. It is a simple, rapid procedure that is easily performed under local anesthesia; and it allows preservation of adequate splenic tissue to safeguard against overwhelming infection. Both splenectomy and PSE are effective in increasing WBC and PLT counts. Although postoperative improvement in WBC and PLT counts by splenectomy can be higher than PSE. PSE is simple and minimally invasive and can be an effective option for patients who are not surgical candidates and for whom splenectomy is contraindicated.

\section{References}

1. Orlando R, Lirussi F, Basso SM, Lumachi F: Splenomegaly as risk factor of liver cirrhosis. A retrospective cohort study of 2,525 patients who underwent laparoscopy. In Vivo 2011; 25: 1009-1012.

2. Qamar AA, Grace ND: Abnormal hematological indices in cirrhosis. Can J Gastroenterol 2009; 23: 441-445.

3. Shah R, Mahour GH, Ford EG, Stanley P: Partial splenic embolization. An effective alternative to splenectomy for hypersplenism. Am Surg 1990; 56: 774-777.

4. Kojouri K, Vesely SK, Terrell DR, George JN: Splenectomy for adult patients with idiopathic thrombocytopenic purpura: A systematic review to assess long-term platelet count responses, prediction of response, and surgical complications. Blood 2004; 104: 2623-2634.

5. Winslow ER, Brunt LM: Perioperative outcomes of laparoscopic versus open splenectomy: A meta-analysis with an emphasis on complications. Surgery 2003; 134: 647-653.

6. Watanabe $Y$, Horiuchi $A$, Yoshida $M$, Yamamoto $Y$, Sugishita $H$, Kumagi T, et al: Significance of laparoscopic splenectomy in patients with hypersplenism. World J Surg 2007; 31: 549555.

7. N'Kontchou G, Seror O, Bourcier V, Mohand D, Ajavon Y, Castera L, et al: Partial splenic embolization in patients with cirrhosis: Efficacy, tolerance and long-term outcome in 32 patients. Eur J GastroenterolHepatol 2005; 17: $179-184$.

8. Hayashi H, Beppu T, Okabe K, Masuda T, Okabe $\mathrm{H}$, Baba H: Risk factors for complications after partial splenic embolization for liver cirrhosis. Br J Surg 2008; 95: 744-750.

9. Zhu K, Meng X, Qian J, Huang M, Li Z, Guan S, Jiang Z, Shan H: Partial splenic embolization for hypersplenism in cirrhosis: A long-term outcome in 62 patients. Dig Liver Dis 2009; 41: 411-416.

10. Sahin M, Tekin S, Aksoy F, Vatansev H, Seker $M$, Avunduk MC, et al: The effects of splenic artery ligation in an experimental model of secondary hypersplenism. $\boldsymbol{J} \boldsymbol{R}$ Coll Surg Edinb 2000; 45: 148-152.

11. Spigos DG, Jonasson O, Mozes M, Capek V: Partial splenic embolization in the treatment of hypersplenism. AJR Am J Roentgenol 1979; 132: 777-782.

12. Hadduck TA, McWilliams JP: Partial splenic artery embolization in cirrhotic patients. World Journal of Radiology 2014; 6: 160-168.

13. Zhan $\mathrm{XL}$, Ji $\mathrm{Y}$, Wang YD: Laparoscopic splenectomy for hypersplenism secondary to liver cirrhosis and portal hypertension. World Journal of Gastroenterology 2014; 20: 57945800 .

14. Feldman LS: Laparoscopic splenectomy: Standardized approach. World Journal of Surgery 2011; 35: 1487-1495,

15. Gould MK, Garcia DA, Wren SM, Karanicolas PJ, Arcelus JI, Heit JA, et al: Prevention of VTE in nonorthopedic surgical patients: Antithrombotic therapy and prevention of thrombosis. Chest 2012; 141: e227S-e277S.

16. Kadir S: Current Practice of Interventional Radiology, B. C. Decker Incorporated 1991.

17. Zhu K, Meng X, Qian J, Huang M, Li Z, Guan S, et al: Partial splenic embolization for hypersplenism in cirrhosis: A long-term outcome in 62 patients. Dig Liver Dis 2009; 41: 411-416. 